\title{
Lung transplantation during COVID-19 outbreak in mainland China
}

\author{
Chun-Rong Ju, Wei-Jie Guan, Jian-Xing He \\ Guangzhou Institute of Respiratory Health, First Affiliated Hospital of Guangzhou Medical University, Guangzhou, China \\ Correspondence to: Jian-Xing He. Guangzhou Institute of Respiratory Health, First Affiliated Hospital of Guangzhou Medical University, Guangzhou \\ 510120, China. Email: hejx@vip.163.com.
}

Submitted May 15, 2020. Accepted for publication Aug 19, 2020.

doi: 10.21037/jtd-20-1948

View this article at: http://dx.doi.org/10.21037/jtd-20-1948

The coronavirus disease 2019 (COVID-19) outbreak, now a public health emergency of international concern, has caused a devastating impact on the world (1). According to the latest estimates, the fatality rate reached to $5.6 \%$ in mainland China. Solid organ transplant (SOT) recipients, mostly the immunosuppressed hosts, are particularly susceptible to the infection of various potentially pathogenic microorganisms including respiratory viruses. More attention should be paid because lung transplant recipients might be more susceptible than any other SOT recipients based on our previous experience.

Recently, the patient number undergoing lung transplantation has been steadily increasing in mainland China (2). To enumerate the patients on the waiting list for lung transplantation and elucidate the impact of COVID-19 outbreaks on the lung transplant recipients, we have collected the related data in mainland China. Of the 30 patients with COVID-19 who have undergone non-lung SOT procedures (3-5), only one lung transplant recipient who was a 65 -year-old male diagnosed as having COVID-19. At the sixth day after symptom onset, he was diagnosed COVID-19. He died of rapidly progressing respiratory failure 2 hours later before being admitted to ICU, while all his family members survived. Table 1 and Figure $1 A$ show the detailed information and computed tomography (CT) findings of the patient.

Furthermore, there has been only one lung transplant recipient who has been diagnosed as having COVID-19 since the outbreak (dating back to December 2019). One reason for the low incidence among lung recipients is that the number of lung transplant recipients was significantly lower than that of other SOT recipients and the general population. The other reason should be attributed to the greater awareness of self-protection, intensive education by the Chinese transplant experts, and online outpatient consultation platform. These strategies have been shown to be effective because none of the lung recipient had been diagnosed as having COVID-19 outside of Wuhan city $(6,7)$.

Lung donation and transplantation in the epicenter were different from those in non-epicenters. Figure $1 B$ shows the number of lung transplantation performed in several centers in mainland China. The total number of lung transplant surgeries performed was 50 during the epidemic period (from 23rd January to 22nd April) in mainland China, with 11 cases being performed from 23rd January to 22nd February, 14 cases from 23rd February to 22nd March, and 25 cases from 23rd March to 22nd April. The mean age of recipients was 46.0 (interquartile range, 28.0-63.5) years. The underlying disease for lung transplantation included idiopathic pulmonary fibrosis (IPF), chronic obstructive pulmonary disease (COPD), connective tissue disease related interstitial lung disease (CTD-ILD), bronchiectasis, pneumoconiosis, pulmonary arterial hypertension (PAH) and other causes (Figure 1C). None of the donors, recipients or medical staff suffered from COVID-19. There were several specific guidelines for organ transplantation during the COVID-19 outbreak in China, and the transplant staff adhered strictly to the guidelines at every step $(8,9)$. As indicated in Figure 1C, patients with IPF were the major recipients in mainland China, which was consistent with the International Society for Heart and Lung Transplantation Guidelines on the transplantation strategy. Unfortunately, given the surge of laboratory-confirmed cases, the high risk of infection, the shortage of medical resources as well as the burnout of staff in the ICU, the organ donation and lung 
Table 1 Clinical characteristics of the lung transplant recipient with COVID-19

\begin{tabular}{|c|c|}
\hline Lung transplant recipient & Information \\
\hline Gender & Male \\
\hline Age & 65 years old \\
\hline Primary indication & Idiopathic pulmonary fibrosis \\
\hline Type of transplant & Right single lung transplant \\
\hline Range of the follow-up period & Every 6 to 7 weeks if stable \\
\hline Immunosuppressant & Tacrolimus, mycophenolate mofetil and methylprednisolone \\
\hline Comorbidities & Diabetes mellitus, chronic kidney disease \\
\hline Date of onset & January $25^{\text {th }} 2020$ \\
\hline Epidemiology & Exposure to his daughter who was diagnosed COVID-19 on January $20^{\text {th }} 2020$ \\
\hline Main symptoms & Mild fever $\left(<38.0^{\circ} \mathrm{C}\right)$, chills, cough, shortness of breath \\
\hline RT-PCR results for SARS-CoV-2 & Negative on January $29^{\text {th }} 2020$ (throat swab); positive on January $31^{\text {st }} 2020$ (throat swab) \\
\hline CT findings & Diffuse ground-glass opacities (Figure 1A) \\
\hline Treatment & Ganciclovir $0.3 \mathrm{~g} / \mathrm{d}$ intravenously and oral moxifloxacin $0.4 \mathrm{~g} / \mathrm{d}$ (January $29^{\text {th }}$ to $31^{\text {st }}$ ) \\
\hline Outcome & Died 2 hours after admission (January $31^{\text {st }} 2020$ ) \\
\hline
\end{tabular}

CLAD, Chronic lung allograft dysfunction; RT-PCR, real time polymerase chain reaction.

transplant surgery had been totally ceased in the epicenters such as Wuhan city since 23rd January, 2020.

In light of the substantially decreased number of new cases with COVID-19, it would be possible to resume lung transplantation for patients with life-threatening respiratory failure throughout mainland China. The resumption of normal daily activities has raised the concern of the potentially new infections from asymptomatic patients (including the donors). To ensure safety, the potentially eligible donors must undergo bronchoscopy, blood and fecal test to preclude the infection with COVID-19, the negative PCR result for SARS-CoV-2 must be confirmed before the organ was procured. For lung transplant recipients during hospitalization, the detection for SARS-CoV-2 should be done routinely at least every week if they are stable, including those who undergo pulmonary rehabilitation in the early stage post lung transplantation. However, if they are unstable or suspected to be infected with SARS$\mathrm{CoV}-2$, the test should be performed timely or frequently. For the recipients who are stable and at home, COVID-19 tests would not be mandatory unless they have new-onset symptoms and are suspected to be infected with SARS$\mathrm{CoV}-2$. Attention should also be paid to the deviation from clinically stable conditions among the recipients who were managed at home, because their symptoms were usually non-specific. Taking into account the observation of the lung recipient who died two hours after hospitalization, we recommend intensive medical observations with a much greater frequency for patients highly suspected of having COVID-19. Notably, the lung transplant recipient initially tested negative to SARS-CoV-2 from the throat swab after symptom onset, while his symptom aggravated and the viral assays became positive two days later. Thus, patients with similar presentation are recommended to be hospitalized and placed under close medical observation. The detection for SARS-CoV-2 should be performed every 1-2 days for the throat swab in suspected cases. However, bronchoalveolar lavage fluid (BALF) might be considered if a recipient was highly suspected of suffering from COVID-19 despite repeated negative findings of the throat 


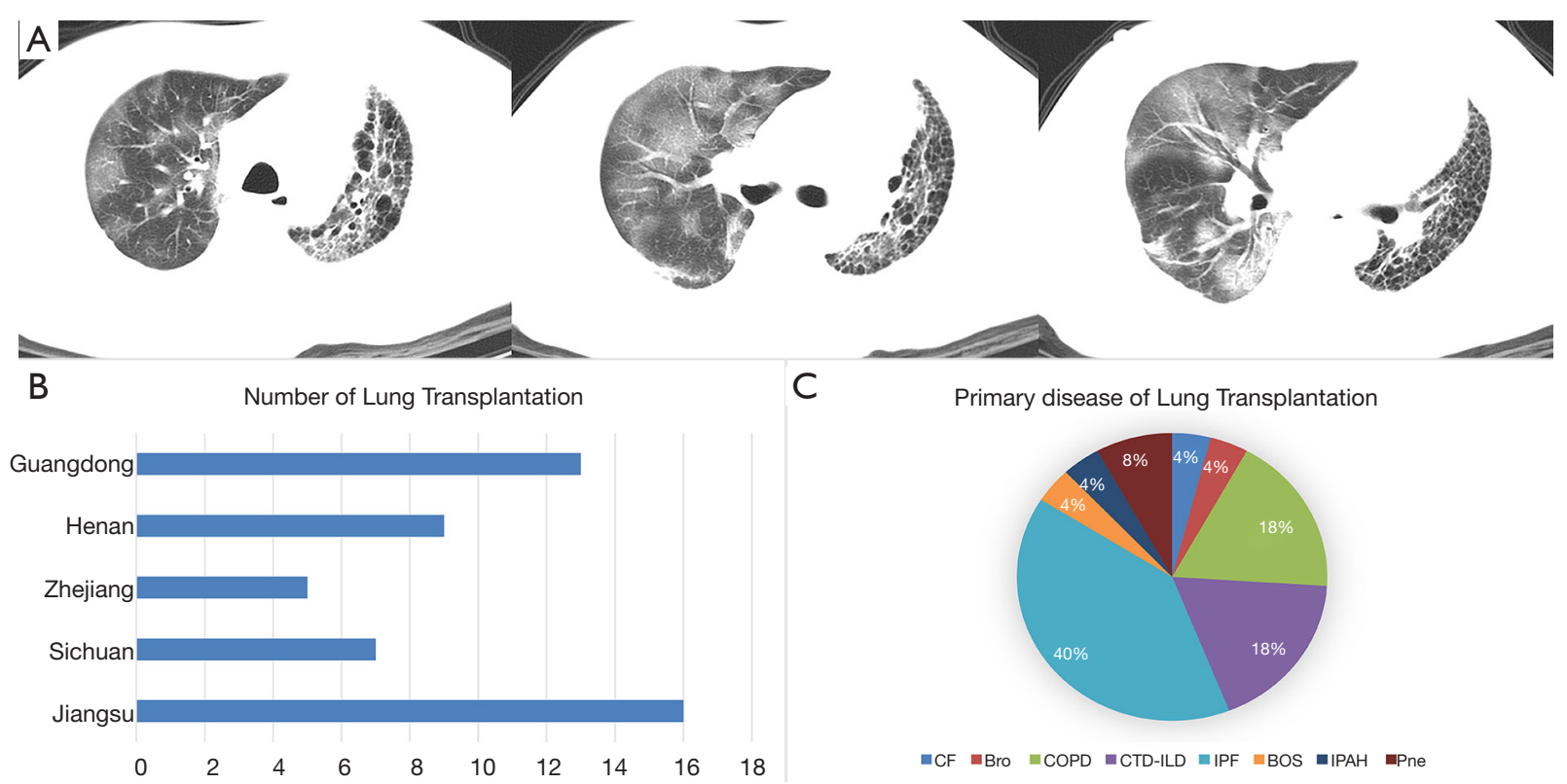

Figure 1 CT manifestations of the lung transplant recipient with COVID-19 and lung transplant surgeries in China during epidemic period. (A) Thoracic CT images of the lungs in a right single lung transplant recipient with COVID-19. The figure shows diffuse groundglass opacities, which are more prominent in the right lung. Reticular pattern and honeycombing are also seen in the left lung. (B) The number of lung transplantation performed in several centers during the outbreak of COVID-19 in mainland China. (C) The underlying diseases for lung transplantation during the COVID-19 outbreak in mainland China. Bro, bronchiectasis; BOS, obliterative bronchiolitis syndrome; COPD, chronic obstructive pulmonary disease; CTD-ILD, connective tissue disease related lung interstitial disease; IPAH, idiopathic pulmonary arterial hypertension; IPF, idiopathic pulmonary fibrosis; Pne, pneumoconiosis.

swab.

Maintenance therapy is crucial to SOT recipients after transplantation. Thus, adjustment for the dosage of immunosuppressants is crucial aside from the background therapy for SOT recipients with COVID-19. Based on the experience on the treatment for COVID-19 patients who had undergone SOT in China (3), we suggest the following strategies for lung recipients suffering from COVID-19: (I) temporally withhold anti-metabolic medication; (II) decrease the dose of calcineurin inhibitors to a certain degree; (III) administer low-dose methylprednisolone or steroids therapy. The strategies had been reported to be effective maintaining the graft function and suppressing the excessive airway inflammatory responses after secondary infections.

Currently, there has been a sharp reduction in the new cases in mainland China. The transplant program was resumed, after the cessation for about 76 days (from 23rd January to 8th April) in the epicenters. The experience might serve as the exemplar for guiding the lung transplantation during the epidemic outbreak.

\section{Acknowledgments}

Funding: The study was supported by the Founding from The State Key Laboratory of Respiratory Disease (SKLRD-QN-201710), the Natural Science Founding of Guangdong Province (2018A030313107), and the Founding from Guangzhou Institute of Respiratory Health (2019GIRHZ04).

\section{Footnote}

Provenance and Peer Review: This article was a free submission to the journal. The article was sent for external peer review.

Peer Review File: Available at http://dx.doi.org/10.21037/jtd20-1948

Conflicts of Interest: All authors have completed the ICMJE uniform disclosure form (available at http://dx.doi. org/10.21037/jtd-20-1948). JXH serves as an unpaid 
Executive Editor-in-Chief of Fournal of Thoracic Disease. The authors have no other conflicts of interest to declare.

Ethical Statement: The authors are accountable for all aspects of the work in ensuring that questions related to the accuracy or integrity of any part of the work are appropriately investigated and resolved.

Open Access Statement: This is an Open Access article distributed in accordance with the Creative Commons Attribution-NonCommercial-NoDerivs 4.0 International License (CC BY-NC-ND 4.0), which permits the noncommercial replication and distribution of the article with the strict proviso that no changes or edits are made and the original work is properly cited (including links to both the formal publication through the relevant DOI and the license). See: https://creativecommons.org/licenses/by-nc-nd/4.0/.

\section{References}

1. Chen J. Pathogenicity and transmissibility of 2019-nCoV-A quick overview and comparison with other emerging viruses. Microbes Infect 2020;22:69-71.

2. $\mathrm{Hu} \mathrm{C}$, Chen $\mathrm{W}, \mathrm{He} \mathrm{J}$, et al. Lung transplantation in China between 2015 and 2018. Chin Med J 2019;132:2783-9.

3. Zhu L, Gong N, Liu B, et al. Coronavirus Disease 2019 Pneumonia in Immunosuppressed Renal Transplant

Cite this article as: Ju CR, Guan WJ, He JX. Lung transplantation during COVID-19 outbreak in mainland China. J Thorac Dis 2020;12(10):6132-6135. doi: 10.21037/jtd-20-1948
Recipients: A Summary of 10 Confirmed Cases in Wuhan, China. Eur Urol 2020;77:748-54.

4. Zhang H, Chen Y, Yuan Q, et al. Identification of Kidney Transplant Recipients with Coronavirus Disease 2019. Eur Urol 2020;77:742-7.

5. The Transplantation Society. Mortality rates in Transplant recipients infected with SARS-CoV-2 and diagnosed as having COVID-19. (software). 2020 April (cite 2020 May 12). Available online: https://tts.org/index. php?option=com_content\&view=article $\&$ id $=696 \&$ Item $\mathrm{id}=115$

6. Ju CR, Li N, Qiu T, et al. Clinical characteristics of novel coronavirus pneumonia in organ transplant recipients and management strategy during the epidemic (1st edition). Organ Transplant 2020;11:185-93.

7. Ju CR, Lian QY, Zhang JH, et al. Recommended prophylactic and management strategies for severe acute respiratory: syndrome coronavirus 2 infection in transplant recipients. Chronic Dis Transl Med 2020;6:87-97.

8. Branch of Organ Transplantation of Chinese Medical Association. Guidelines for organ donation and transplantation in China during novel coronavirus pneumonia epidemic. Organ Transplant 2020;11:179-84.

9. Chen S, Zhang WJ, Chen G, et al. Guidelines for diagnosis and treatment of COVID-19-infected organ transplant recipients and the organ transplant surgery. Chin J Transplant 2020;41:E003-3. 